FZMw Jg. 5 (2002) S. 94-110

\title{
Meditationen, Geheimnisse, Zufälle: Messiaens Konzept der langage communicable
}

\author{
von Rebecca Grotjahn
}

Vorbemerkung: Dieser Text ist der Vorabdruck eines Beitrags zum Kölner Symposion Olivier Messiaen. La cité céleste - Das himmlische Jerusalem, das 1998 im Rahmen des Projekts La cité céleste. Olivier Messiaen zum Gedächtnis von der GuardiniStiftung veranstaltet wurde (Projektleitung: Thomas Daniel Schlee) ${ }^{1}$.

$-1-$

Kaum ein Komponist der Neuen Musik ist so beliebt wie Olivier Messiaen. Bevorzugt erklingen seine Werke ausgerechnet in Kirchenräumen, in denen die Darbietung von Musik des 20. Jahrhunderts nicht unbedingt zu den Selbstverständlichkeiten gehört. Wie ist es zu erklären, daß Messiaens Werke nicht annähernd so starke Ressentiments auslösen wie die geistlichen Werke eines Karlheinz Stockhausen, Dieter Schnebel oder auch des - doch weitaus "traditionelleren" - Arnold Schönberg? Sicherlich sind es zum einen bestimmte Eigenschaften seiner Musik, die einer nichtspezialisierten Hörerschaft entgegenommen: die sprichwörtliche Farbigkeit des Klangs und der Harmonik oder die relativ einfachen, wiederholungsreichen Formanlagen. Von entscheidender Bedeutung dürfte jedoch ein anderer Grund sein: Der Komponist läßt seinen Hörern Hilfen zukommen in Form zahlreicher Verbaltexte, die den Stücken als Erklärung und Interpretation beigegeben sind. Kaum ein Werk ohne ausführliche "note de l'auteur", in der kompositionstechnische Details erläutert, vor allem aber der theologische Gehalt der Musik dargelegt wird. Darüber hinaus finden sich auch in den Notentexten zahlreiche Anmerkungen, die dafür sorgen, daß die Hinweise der Vorworte der Musik korrekt zugeordnet werden ${ }^{2}$.

$\overline{1}$

Ein von Elmar Budde, Thomas Daniel Schlee und Christine Wassermann Beirão herausgegebener Bericht über das Kölner Symposion 1998 wird im Laaber-Verlag erscheinen. Bereits vorgelegt wurde der Band Olivier Messiaen. La Cité céleste - Das himmlische Jerusalem. Über Leben und Werk des französischen Komponisten, hrsg. von Thomas Daniel Schlee und Dietrich Kämper, Köln 1998.

2 Zur Rezeption Messiaens in der evangelischen Kirchenmusik siehe auch: Rebecca Grotjahn, Theologische Musik? Neue Musik? Messiaen, die evangelische Kirchenmusik und die Moderne, in: Le Sacre. Musik - Ritus - Religiosität; Biennale Neue Musik Hannover 2001, hrsg. von Hinrich Bergmeier, Saarbrücken 2001, S. 143-156. 
Diese Erläuterungen und Kommentare dürften nicht zuletzt auch für die große Menge der Messiaens Werken gewidmeten Literatur verantwortlich sein. Ein großer Teil dieser Schriften ist von der Überzeugung getragen: "Es gibt keine wesentlicheren Auskünfte über Olivier Messiaen als von Olivier Messiaen." ${ }^{, 3}$ So sieht Aloyse Michaely in den Texten des Komponisten die Grundlage für die korrekte Ausdeutung seiner Werke: Sie "ermöglichen es, die konkrete theologische Aussage eines Werkes so genau zu bestimmen, daß kaum Raum für Spekulationen bleibt" ". Der Autor sieht seine Aufgabe darin, das "Vokabular", das sich Messiaen geschaffen habe, um seine Ideen auszudrücken, "systematisch zu ordnen und darzustellen", vielen anderen Texten über den Komponisten - zu einer opulenten und aufgrund des Arbeitsaufwandes bewundernswerten Aufzählung der Einzelheiten: der verwendeten Motive, der benutzten Kompositionstechniken und der "Übersetzungen" in theologische Aussagen, entsprechend den Vorgaben des Komponisten.

$$
-3-
$$

Es ist an der Zeit, daß sich die Messiaen-Forschung aus der inneren Abhängigkeit von ihrem Forschungsgegenstand befreit und die Texte des Komponisten selbst zum Thema der Reflexion macht. Dies soll im folgenden am Beispiel der Méditations sur le mystère de la Sainte Trinité geschehen. Zunächst ist der Text des Vorwortes auf seine Triftigkeit zu befragen. $\mathrm{Zu}$ überprüfen ist weiterhin das Verhältnis von Vorwort und Musik: Geht die Musik auf in dem im Vorwort Gesagten, geht sie womöglich darüber hinaus oder gar daran vorbei? Diese Frage läßt sich nicht ohne musikalische Analyse beantworten - eine Analyse freilich, die ihre Fragen selbst stellt anstatt nur die Bemerkungen des Vorwortes zu bestätigen.

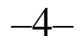

Das Vorwort zu den Méditations ist nicht, wie die meisten Vorworte zu Werken Messiaens, überschrieben mit "Note de l'auteur" oder "Préface", sondern es trägt eine auf den Inhalt vorausweisende Überschrift: Le langage communicable. Der Komponist verspricht hier also eine Art Abhandlung zum Thema Sprache und gibt damit einen - im

Ingrid Hohlfeld-Ufer, Die musikalische Sprache Olivier Messiaens, dargestellt an dem Orgelzyklus "Die Pfingstmesse", Duisburg 1978, S. 6.

Aloyse Michaely, Die Musik Olivier Messiaens. Untersuchungen zum Gesamtschaffen, Hamburg 1987 (Hamburger Beiträge zur Musikwissenschaft. Sonderband), S. 6.

Vgl. ebd. 
Vergleich zu einem einfachen Vorwort - höheren Maßstab für den Anspruch des Textes vor. Als Übersetzung für "Le langage communicable" hat sich in deutschsprachigen Publikationen die Formulierung "die kommunizierbare Sprache" eingebürgert, die nicht nur grammatikalisch unstimmig ist ${ }^{6}$, sondern auch die Differenz zwischen der französischen und der deutschen Vokabel verkennt. Für "Le langage communicable" hat sich in deutschsprachigen Publikationen die Übersetzung "die kommunizierbare Sprache" eingebürgert ${ }^{7}$ - ein ungrammatisches Begriffsungetüm, das keinen Sinn ergibt Das französische Wort "communicable" ist mit "übertragbar" oder "mitteilbar" zu übersetzen, und eben darum geht es in dem Konzept der langage communicable: um die Übertragung eines Bedeutungsgehaltes von einem Zeichensystem ins andere, um die Mitteilung einer Nachricht in einer neu konzipierten Sprache. Messiaens Thema ist nicht der Kommunikationsaspekt der Sprache, sondern das Verhältnis von Zeichen und Bedeutung. Der erste Satz des Textes: “Les diverses langues connues, sont, avant tout, des instruments de communication" " wäre zu übersetzen: Die verschiedenen bekannten Sprachen sind, vor allem, Instrumente - nicht: der Kommunikation, sondern: - der Übermittlung oder Mitteilung. Im allgemeinen, so Messiaen, verwende Sprache vokale Zeichen, vorstellbar seien jedoch auch Bilder, Farben oder Düfte als Zeichensysteme. Sein Beispiel für eine auf dem Tastsinn beruhende Sprache allerdings leidet unter einer gedanklichen Unschärfe, denn das Braille-Alphabet überträgt nicht Begriffe in ertastbare Zeichen, sondern nur die einzelnen Buchstaben des Alphabets, das ja selbst keine Sprache ist. Alle genannten Sprachen beruhen, so Messiaen weiter, auf vorausgehenden Konventionen: "Il est convenu que ceci exprimera cela”. Eben diese Möglichkeit besitze die Musik jedoch nicht: Sie könne zwar Gefühle und Seelenzustände ausdrücken, aber es fehlten Konventionen, die die genaue Bedeutung musikalischer "Ausdrücke" festlegten.

6 Das Suffix “-bar" steht für "was gemacht werden kann” und kann somit nur mit dem Stamm eines passivfähigen Verbs verbunden werden (vgl. Ulrich Engel, Deutsche Grammatik. 2., verbesserte Auflage, Heidelberg 1988, S. 579). Das Verb "kommunizieren” ist nicht passivfähig; die Umformung des Ausdrucks "kommunizierbare Sprache" in "Sprache, die kommuniziert werden kann", ist unmöglich.

Nachdenklich stimmt, daß sich die Formulierung "kommunizierbare Sprache" halten konnte, obwohl sich schon das Sprachgefühl dagegen widersetzt. Ich vermute einen Zusammenhang mit den Versuchen mancher Messiaen-Exegeten, die Musik des Komponisten als eine Art Geheimsprache aufzufassen, die nur Eingeweihten zugänglich ist.

7 Vermutlich erstmals von Almuth Rößler in Sieglinde Ahrens, Hans Dieter Möller und Almuth Rößler, Das Orgelwerk Messiaens, Duisburg 1968, S. 56.

Die hier und im folgenden nicht einzeln nachgewiesenen Zitate entstammen dem Vorwort zu Olivier Messiaen, Méditations sur le mystère de la Sainte Trinité pour Orgue, Paris: Editions Musicales Alphonse Leduc, 1973. 
Mit dieser Auffassung von der Differenz zwischen Wortsprache und Musik als "Sprache des Gefühls" greift Messiaen einen musikästhetischen Topos auf, für den er Richard Wagner als Gewährsmann anführt - allerdings nicht, ohne ihn gewaltig mißzuverstehen: Wagner habe das Leitmotiv als langage communicable erfunden, und auch hier regelten Konventionen die Bedeutung der Motive. Indem er die Leitmotive sogar explizit als "Begriffe" ("notions") bezeichnet, verkehrt Messiaen den Begriff ins Gegenteil, hat bei Wagner der Orchestersatz - dessen Teil die Leitmotive sind - doch gerade die Funktion, das "Unaussprechliche", die eben nicht präzise in Worten faßbaren Seelenregungen, Ahnungen und Beziehungen der Figuren zum Ausdruck zu bringen ${ }^{9}$. Das Mißverständnis zeigt um so deutlicher die Intentionen Messiaens: Er zielt auf eine eindeutige musikalische Begriffssprache, die er im weiteren Verlauf des Textes - nach einem Exkurs über die Sprache der Engel - zu entwickeln beginnt.

$$
-6-
$$

Allerdings ist dieses Konzept einer musikalischen Sprache von vornherein ausgesprochen problematisch, da schon der Ausgangspunkt unlogisch ist. Wie bereits im Zusammenhang mit dem Braille-Alphabet deutlich wurde, versäumt es der Autor, zwischen den Begriffen "Sprache" und "Schrift" zu unterscheiden:

\section{“[...] j’ai cependant essayé, par jeu, et pour renouver ma pensée, de trou- ver une sorte de langage musical communicable. \\ J'ai d'abord voulu faire un alphabet musical au moyen duquel je pourrai transcrire des mots (des mots français, tout nä̈vement, parce que je suis français)." $" 10$}

$$
-7-
$$

Die Übertragung französischer Worte in ein musikalisches Alphabet ist freilich keine Übersetzung in eine andere Sprache, ebensowenig wie z. B. die Transkription eines in kyrillischen Buchstaben geschriebenen russischen Satzes in die lateinische Schrift.

\footnotetext{
9 Vgl. z. B. Oper und Drama, in: Richard Wagner, Dichtungen und Schriften, hrsg. von Dieter Borchmeyer, Frankfurt am Main 1983, Bd. 7, bes. S. 308ff. oder Zukunftsmusik, a. a. O., Bd. 8, bes. S. 70ff. Vgl. dazu auch Carl Dahlhaus, Wagners Konzeption des musikalischen Dramas, Regensburg 1971, bes. S. 21f. lisch mitteilbare Sprache zu finden. - Zunächst wollte ich ein musikalisches Alphabet machen, mittels dessen ich die Worte übertragen könnte (französische Worte - naiverweise, weil ich Franzose bin). (Übs.: R. G.)
} 
Messiaen ordnet nun jedem Buchstaben des Alphabets eine Tonqualität, eine bestimmte Oktavlage und eine Tondauer zu. Darüber hinaus werden bestimmte Formeln festgelegt, die die jeweils verwendeten grammatikalischen Fälle anzeigen, so daß Pronomina, Konjunktionen und Präpositionen, aber auch Deklinations- und Konjugationsendungen der Worte aus der Textvorlage nicht Buchstabe für Buchstabe in das musikalische Alphabet übertragen werden müssen. Hinzu kommen schließlich Formeln für die Wörter "être" und "avoir" einschließlich ihrer Ableitungen - die eine die Umkehrung der anderen - sowie ein "thème de Dieu". Das auf diese Weise entstandene Tonmaterial bestimmt zwar nicht alle Dimensionen des Werkes, denn nur einige Abschnitte sind in der langage communicable verfaßt, und auch in diesen tritt jeweils noch eine Begleitstimme hinzu, die nach anderen Regeln gestaltet ist. Als vorgeordnetes Material ist es im Prinzip jedoch vergleichbar mit den Modi, die Messiaen in seinem Klavierstück Mode de valeurs et d'intensités verwendet, oder auch den Reihen, die serieller Musik zugrunde liegen. Der Tonvorrat ist begrenzt, und die Reihenfolge der Töne ist determiniert durch die Textgrundlage des Werkes.

$$
-8-
$$

Damit steht das Konzept der langage communicable freilich im Gegensatz zu derjenigen Musikauffassung, auf die sich Messiaen zu Beginn seines Textes bezogen hatte. Der Topos von der Musik als Sprache, der ja auf eine lange musikgeschichtliche Tradition zurückblicken kann, verbindet sich stets mit der Überzeugung, daß die musikalische "Sprache" zwar nicht eindeutig in Wortsprache übersetzbar, gleichwohl jedoch von jedem Hörer unmittelbar verständlich und nachvollziehbar ist und somit gar keiner Übereinkünfte über die Bedeutung der "Zeichen" bedarf. Er basiert auf dem Begriff einer musikalischen Logik, die sich in motivisch-thematischen Beziehungen und funktionsharmonischen Entwicklungen vollzieht. Ulrich Siegele sieht den Verlust dieser Sprachhaftigkeit von Musik als den entscheidenden Einschnitt in der Musikgeschichte des 20. Jahrhunderts an. Die serielle Musik bricht mit traditionellem Musikdenken, indem sie das musikalische Material für jedes Werk individuell vorordnet und so den Anspruch der "potentiellen Allgemeinverständlichkeit" aufgibt: "An die Stelle von Musik als Mitteilung, von Musik als Sprache tritt die Vorstellung von Musik als Spiel auf der Grundlage der Kombinatorik." 11

$11 \quad$ Vgl. hierzu Ulrich Siegele, Entwurf einer Musikgeschichte der sechziger Jahre, in: Die Musik der sechziger Jahre. Zwölf Versuche, hrsg. von Rudolph Stephan (Veröffentlichungen des Instituts für Neue Musik und Musikerziehung, Bd. 12), Mainz 1972, S. 9-25; das Zitat: S. $14 f$. 
In Messiaens langage communicable wird ein Begriff aus der Wortsprache nicht durch ein unmittelbar nachvollziehbares Tonsymbol ausgedrückt. Vielmehr kommt es durch die Verwendung des Tonalphabets gerade zu einer für den Hörer als willkürlich empfundenen Zuordnung von Begriffsinhalten und Tonfolgen. Diese Sprache ist eben nicht allgemeinverständlich, im Gegenteil: Es entstehen Tonfolgen, die jeder Nachvollziehbarkeit oder "Logik" entbehren, solange die hier geltenden "Spielregeln" nicht bekannt sind. Dieser Umstand hat auch Irritation ausgelöst. So berichtet Almuth Rößler über eine Diskussion im Rahmen des 2. Düsseldorfer Messiaen-Festes (1972): "Ein junger Mann aus dem Publikum stellt eine ziemlich aggressive Frage etwa des Inhaltes, daß die Benutzung eines Ton-Alphabets kein kompositorischer Akt, sondern eher ein simples Ausnutzen des Zufalls sei." ${ }^{12}$ Dem ist zuzustimmen, insofern die Töne Funktionen der Materialvorordnung und keine Früchte der "Inspiration" sind. Damit jedoch ist die langage communicable eine von vielen Methoden des Komponisten, die musikalische Erfindung gleichsam in den neutralen Raum außerhalb des komponierenden Subjektes zu verlegen ${ }^{13}$. Sie ist insoweit vergleichbar den Werken bzw. Passagen, die der Natur sorgfältig abgelauschte Vogelrufe verwenden, aber auch den zahlreichen Versuchen mit oft relativ simplen, jedoch strikt eingehaltenen Konstruktionsprinzipien, die den Komponisten seit Beginn der vierziger Jahre beschäftigten ${ }^{14}$.

$$
-10-
$$

Die Umsetzung eines Textes in die langage communicable sei am Beispiel des 1. Satzes der Méditations genauer betrachtet. Textgrundlage für dessen vierten Abschnitt bilden einige Sätze aus der Summa theologica des Thomas von Aquin in französischer Übersetzung. Diese Sätze werden entsprechend den im Vorwort dargelegten Verfahrensweisen umgeformt: "Kleine Worte" entfallen, Kasus, Negationen sowie die Verben "haben" und "sein" werden durch bestimmte Formeln ausgedrückt. Auch das Wort "Pére" drückt der Komponist nicht in Tonbuchstaben aus, sondern durch eine aus der "Sein"Formel abgeleitete Tonfolge (die jedoch im Vorwort noch nicht vorgestellt wurde).

\footnotetext{
13 Almuth Rößler, Beiträge zur geistigen Welt Olivier Messiaens, Duisburg 1984, S. 58. Vgl. auch Ernst, die die Langage communicable als "Hilfsmittel der "inventio" bezeichnet, das "Elemente des Soggetto "cavato" wie auch der Aleatorik" vereine. (Karin Ernst, Der Beitrag Olivier Messiaens zur Orgelmusik des 20. Jahrhunderts, Freiburg 1980 [HochschulSammlung Philosophie Musikwissenschaft, Bd. 1], S. 154.)

Z. B. Canon rythmique de plus en plus serrés, Canon par ajout du point, Agrandissements asymétriques, Valeurs progressivement ralenties oder Valeurs progressivement accélerées usw., die sich häufig in Werken wie den Visions de l'Amen oder den Vingt regards sur l'Enfant-Jésus finden.
} 
Übersetzt man den Text aus der langage communicable zurück ins Französische, so ergibt sich ein vollkommen kryptischer Text (in eckigen Klammern wird die Bezeichnung der Formeln wiedergegeben, in Großbuchstaben die nach Messiaens Regelsystem ermittelten Tonbuchstaben):

\author{
[Père] [Akkusativ/Dativ] PERSONNES PROCÉDANT [Abla- \\ tiv/Genitiv/Lokativ] [Père] [Haben] PATERNITÉ SPIRATION [Père] [Ak- \\ kusativ/Dativ] PRINCIPE [Negation] PRINCIPE [Haben] [Negation] [Sein] \\ [Genitiv/Ablativ/Lokativ] AUTRE [Sein] [Haben] INNASCIBILITÉ [Sein] \\ INENGENDRÉ [Père]
}

Zieht man die Anmerkungen Messiaens im Notentext heran, wo über den Formeln die jeweils gemeinte Bedeutung angegeben wird, läßt sich ein sprachlich sinnvoller Text lesen (in runden Klammern wird die Übertragung der Formeln angegeben):

[Père] (vers les) PERSONNES PROCEDANT (du) [Père] (il a) PATERNITÈ SPIRATION [Père] (vers le) PRINCIPE (sans) PRINCIPE (il a) (ne pas) (être) (d'un) AUTRE (c'est) (avoir) INNASCIBILITÉ (être) INENGENDRÉ Père

Die Bedeutung dieser Worte jedoch bleibt ohne Kenntnis der Textgrundlage - die im Notentext vor Beginn des 1. Satzes zitiert wird - nach wie vor unklar; diese konfrontiert den Leser ihrerseits mit erheblichen Verständnisproblemen, die ohne theologische Vorkenntnisse kaum zu bewältigen sind:

"Par rapport aux personnes qui procèdent de lui, le Père notifie ainsi: paternité et spiration; en tant que 'Principe qui n'a pas de principe', il se notifie ainsi: il n'est pas un autre: c'est là précisement la propriété d'innascibilité, désignée par le nom d'Inengendré."

Durch die Übertragung in die langage communicable wird also eine ohnehin schwierige Mitteilung weiter verschlüsselt. Beim bloßen Hören ohne Notentext wäre sie überhaupt nicht verständlich, aber auch mit den dem Notentext hinzugefügten Hilfen bleibt ihr 
Sinn weitgehend unklar. Die langage communicable zielt gerade nicht auf das Verstandenwerden, sondern dient der Verdunkelung ${ }^{15}$. Der "Empfänger" weiß, daß "gesprochen" wird, er kennt sogar womöglich die Verfahrensweisen der Sprache, aber der Sinn der Mitteilung bleibt ein Geheimnis.

$$
-13-
$$

Als ein an der Tradition orientierter, ja geradezu romantischer Komponist erscheint Messiaen nur in den allgemein gehaltenen Anfangspassagen seines Textes ${ }^{16}$. Die Verwendung des Topos "Musik als Sprache” bedeutet hier einen Rückgriff auf ein nur unzureichend reflektiertes Klischee, das sich mit der modernen Konzeption der langage communicable nicht vereinbaren läßt. Die Widersprüchlichkeit innerhalb eines und desselben Textes allerdings ist typisch für Messiaen.

$$
\begin{gathered}
\text { II } \\
-14-
\end{gathered}
$$

Den oben zitierten Vorwurf der vom "Zufall” bestimmten Gestaltung hat der Komponist mit dem Hinweis beantwortet, daß immerhin bei der Gestaltung der Begleitstimmen und der Form kompositorische Entscheidungen zu fällen waren:

"Bei mir waren es die Buchstaben, die unerwartete Töne ergaben, die mich gezwungen haben, Akkorde und Tonkombinationen zu suchen. Der Anfang ist vielleicht vom Zufall bestimmt, aber die Folge ist überhaupt nicht zufällig, sondern überlegt und beabsichtigt. ",17

Dieser Rechtfertigung möchte ich eine weitergehende Hypothese gegenüberstellen: Auch die Hauptstimmen der nach den Prinzipien der langage communicable konstruierten Sätze sind das Ergebnis einer bewußten kompositorischen Arbeit - eine Arbeit,

15 Damit widerspreche ich der Auffassung Josef Häuslers, die Langage communicable rechne "mit einem Hörer, der nicht nur der dem Werk zugrundeliegenden Sprache mächtig wäre, sondern die Gabe des absoluten Gehörs besäße und überdies noch fähig wäre, Tonhöhenverläufe im Moment des Hörens in verbale Zusammenhänge zu übertragen." (Josef Häusler, Versuch über Messiaen, in: Olivier Messiaen. Frankfurt Feste '84. Alte Oper Frankfurt, Frankfurt am Main o. J., S. 6-17, das Zitat: S. 15.) Die Tatsache, daß dies vollkommen "illusorisch" ist (ebd.), zeigt m. E. nicht, daß Messiaen sich unrealistischen Vorstellungen über seine Hörer hingibt, sondern daß sein Konzept gar nicht auf theologische Aufklärung zielt.

Auch das 1936 formulierte Manifest der Gruppe "Jeune France", deren Mitglied Messiaen war, greift romantische Denkweisen auf und wendet sich gegen "mechanistische" Tendenzen, die den nur kurze Zeit später entstandenen Werken Messiaens gleichwohl kaum abzusprechen sind. (Vgl. Serge Gut, Le groupe Jeune France, Paris 1984, S. 16f.)

Gespräch mit Olivier Messiaen am 23. April 1979 in Paris, in: Almuth Rößler, Beiträge (s. Anm. 12), Duisburg 1984, S. 71-124; das Zitat: S. 74f., im Anschluß an einen Vergleich mit Bachschen Choralbearbeitungen. 
die nicht erst im Augenblick der Konzeption des Zeitverlaufes verrichtet wird, sondern die bereits die Vorordnung des Materials betrifft. Das Ton-Alphabet selbst ist bereits mit Blick auf das künftige musikalische Ergebnis hin erfunden.

$$
-15-
$$

Freilich steht diese Erkenntnis im Gegensatz zu der Darstellung des Komponisten im Vorwort der Méditations. Laut Messiaen sind es nämlich äußere, außermusikalische Prinzipien, die die Zuordnung von Buchstaben zu Tönen bestimmen. Die Basis bildet das "alphabet musical allemand", das vorab die Töne A B C D E F G $H$ festlegt. Hiervon werden dann alle anderen Buchstaben entsprechend phonologischen Prinzipien anscheinend völlig folgerichtig abgeleitet ("en groupant les lettres par genres de productions phoniques”). So folgt aus der bereits im “deutschen Tonalphabet” festgelegten Quinte $A-E$ die Quintschrittfolge für sämtliche Vokale:

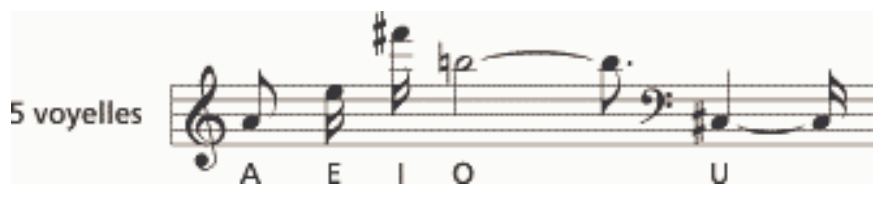

Das $\mathrm{Y}=$ fis " und das $\mathbf{J}=$ fis " leiten sich vom bereits im vorausgehenden Schritt ermittelten I = fis “" ab; als Terzenkette werden die "labiales" P, F, V, M dem bereits festgelegten B zugeordnet.

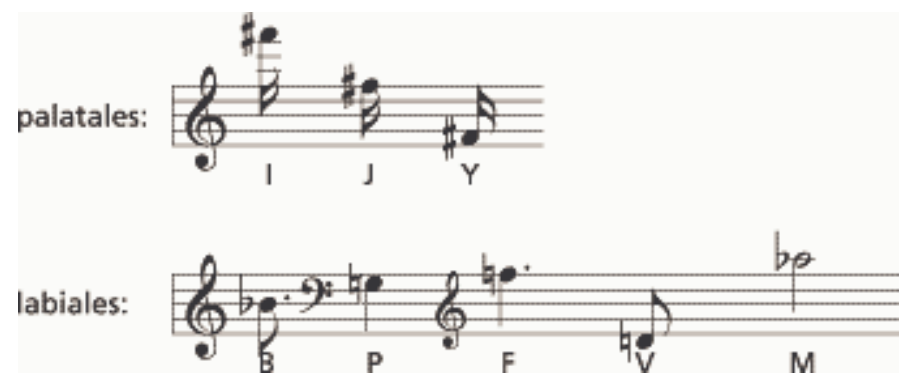

Allerdings erscheint diese Zuordnung zwingender, als sie in Wirklichkeit ist ${ }^{18}$.

$18 \quad$ Vgl. zu den folgenden Ausführungen etwa: Duden. Grammatik der deutschen Gegenwartssprache. 4., völlig neu bearbeitete und erweiterte Auflage. Mannheim, Wien, Zürich 1984, S. 26-58. 
1. Buchstaben sind keine Laute, vielmehr sind ihnen - je nach Sprachsystem - bestimmte Laute zugeordnet. Dabei ist die Verbindung Schriftzeichen - Phonem nur selten eindeutig. Vielen Buchstaben sind mehrere Laute zugeordnet (z. B. S, das als [s] oder als [z] gesprochen wird), andererseits werden einige Buchstaben als Phonemkombination ausgesprochen (z. B. Z als [ts] oder X als [ks]), und schließlich lassen sich zahlreiche Laute nur als Buchstabenkombination aufschreiben (z. B. das [〕], das im Deutschen als Sch, im Französischen meist als Ch geschrieben wird).

$$
-17-
$$

2. Laute lassen sich nach mehreren Kriterien ordnen. In der Phonologie werden zwei Klassifikationskriterien unterschieden: die Artikulationsart und die Artikulationsstelle. Nach dem ersten Kriterium ist z.B. das [p] ein Verschlußlaut (Plosiv), nach dem zweiten ein Bilabiallaut. Messiaen vermengt beide Klassifikationssysteme, wenn sich inmitten der nach dem jeweils artikulierenden Organ geordneten Zeichengruppen (" $p a$ latales, dentales, labiales, linguales”) die Gruppe "sifflantes" (Zischlaute) findet, die die Art der Artikulation angibt.

$$
-18-
$$

3. Messiaen bildet Gruppen nach den bei der Lautbildung beteiligten Organen: Gaumen, Zähne, Lippen, Zunge. In Wirklichkeit sind an der Lautbildung jedoch oft zwei Organe beteiligt, weshalb die Phonetik nicht "Labiale", "Dentale" etc. unterscheidet, sondern Bilabiale, Labiodentale usw. Aufgrund des unzulänglichen Kriteriums "lautbildendes Organ" kommt es bei Messiaen zu Unstimmigkeiten bei der Zuordnung. So spaltet er die Gruppe der Dentale und Alveolaren, die durch Artikulation der Zunge gegen die oberen Schneidezähne oder gegen die Alveolen gebildet werden, in "dentales” (D, T) und "linguales” ( $\mathrm{L}, \mathrm{N})$, während er die phonetisch ebenfalls in diese Gruppe gehörenden Buchstaben S und Z (die Laute [s] und [z]) den "sifflantes" zuordnet.

4. Buchstaben, deren Einordnung - aus welchem Grunde auch immer - Schwierigkeiten bereitet, werden großzügig zu einer Gruppe der "Übrigen” zusammengefaßt: “En ajoutant le $R$, le $W$, le $X . . . "$

$$
-20-
$$

5. Die Zuordnung der Buchstaben C, Q und K zu einer Gruppe “ $C$ dur” [sic] bedeutet einen weiteren Wechsel des Ordungskriteriums, der beinahe wie das selbstironische Eingeständnis des Autors anmutet, mit der phonologischen Klassifikation überfordert zu sein. 
Sinn dieser Anmerkungen ist es nicht, dem Künstler Messiaen den pseudowissenschaftlichen Charakter seiner langage communicable vorzuhalten, auch wenn sie bestätigen, wie angebracht Vorsicht im Umgang mit den Kommentaren des Komponisten ist. Wichtiger jedoch sind die Konsequenzen für die Werkanalyse. Denn die Tatsache, daß sich der Komponist bei der Zuordnung von Tönen und Buchstaben durchaus einige Freiheiten genommen hat - und zwar gerade auch bei häufig auftretenden Buchstaben wie dem $\mathrm{R}$ oder dem $\mathrm{N}$-, weist darauf hin, daß wohl in erster Linie musikalische Kriterien den Ausschlag gegeben haben. Dies gilt erst recht für die völlig freie Festlegung von Oktavlagen und Tondauern.

$$
-22-
$$

Am Beispiel des ersten Satzes der Méditations soll im folgenden untersucht werden, welche kompositorischen Strategien die Zuordnung von Tönen und Buchstaben bestimmt haben. Es handelt sich bei diesem Satz um eine Art Variationenfolge über das in einem ersten Abschnitt einstimmig präsentierte Thema Le Père des étoiles, das sich im Laufe der Variationen immer mehr auflöst. Die Oberstimme des vierten Abschnitts (= 3. Variation) ist in der langage communicable geschrieben; sie zeigt dennoch deutliche Anklänge an das Thema.

$$
-23-
$$

In einem ersten Schritt ist die Häufigkeit der Tonbuchstaben zu bestimmen (siehe Tabelle). Bei der Analyse ist auszugehen von dem für die Umsetzung in die langage communicable präparierten Text, wie er oben zitiert wurde.

Tabelle: Häufigkeit der Tonbuchstaben

\begin{tabular}{|c|c|c|c|c|c|c|c|c|c|}
\hline Tonbuchstabe & E, I, N & P, R & T & A & C, S & O & D & B, G, L, U & $\begin{array}{c}\text { F, H, J, K, M, Q, } \\
\text { V, W, X, Y, Z }\end{array}$ \\
\hline $\begin{array}{c}\text { Häufigkeit des } \\
\text { Auftretens }\end{array}$ & 12 & 8 & 6 & 5 & 4 & 3 & 2 & 1 & 0 \\
\hline
\end{tabular}


Die Häufigkeitsverteilung der Buchstaben ergibt sich aus den Eigenschaften der französischen Sprache. Bei einer Gesamtzahl von 80 Tonbuchstaben verteilen sich $45 \%$ auf die drei Buchstaben $\mathrm{E}$, I und N, die je 12mal vorkommen; weitere 33\% entfallen auf die Buchstaben P, R, T und A, die zwischen 5- und 8mal auftreten ${ }^{19}$. Betrachtet man die diesen häufig verwendeten Buchstaben zugeordneten Dauern und Oktavlagen, so scheint auf den ersten Blick eine Gleichverteilung vorzuliegen, also gerade kein auffälliges Bild. Zwei Töne (E und I) haben sehr kurze Dauern, zwei (N und T) sehr lange, drei (P, A und R) mittlere; zwei Töne befinden sich in sehr hoher Lage (I und R), zwei in tiefer $(\mathrm{P}$ und $\mathrm{T})$, drei in mittlerer.

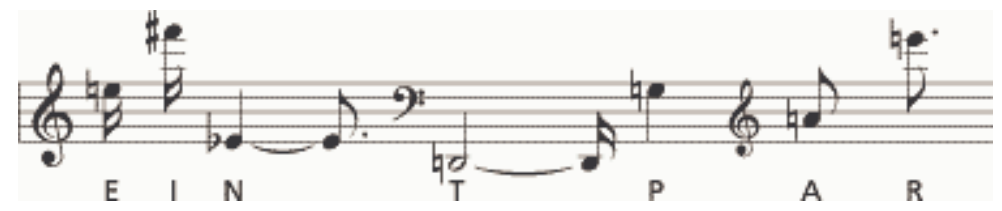

Eine Auffälligkeit im Notentext weist jedoch auf die Motivation der Zuordnung von Tönen, Lagen und Dauern zu den Buchstaben des Textes hin. Der Komponist hat zahlreiche Zäsuren in die Hauptstimme eingefügt, die, bezieht man sie auf die Textgrundlage, die Textworte regelrecht zerreißen. So ergeben sich z. B. die Tonbuchstabenfolgen:

P, ERSO, N, N, ES, P, ROCÉDAN, T (S. 10, II. bis IV. System)

PA, T, ER, N, ITÉSP, IRA, T, IO, N (S. 10, 6. System bis S. 11, 2. System)

Einerseits bedeutet diese Zerstückelung der Worte, daß der oben beschriebene Prozeß der "Verdunkelung" des Textsinns - durch die Übertragung in die langage communicable ohnehin weit fortgeschrittten - noch weiter vorangetrieben wird. Andererseits jedoch ergeben sich durch die Zäsurierung musikalische Motive, die vom Gestus her

19 Die Analyse der - weitaus kürzeren - Texte, die im 3. und 7. Satz des Werkes verwendet werden, bestätigt die hier genannten Tendenzen. Nr. 3 hat das E als häufigsten Tonbuchstaben (6mal), es folgen I und $\mathrm{N}$ (je 3mal) sowie $\mathrm{S}$ und $\mathrm{T}$ (je 2mal). In Nr. 3 sind A und $\mathrm{E}$ am häufigsten (je 3mal), je 2mal erklingen C, N und R. Summiert man die Werte aller drei Langage communicable-Sätze, so ergibt sich bei insgesamt 124 Tonbuchstaben die Rangfolge: E (21mal), N (17), I (16), R (11), A, P und T (je 9mal).

$\mathrm{Zu}$ vergleichen waren schließlich die Buchstabenhäufigkeiten der tatsächlich in Tonbuchstaben umgesetzten Worte mit denen der unpräparierten Textvorlage, also dem unveränderten Thomas von Aquin-Text in französischer Übersetzung. Bei insgesamt 213 Buchstaben waren die sieben häufigsten auch hier E (35mal), I (28), N (24), P (18), A, R und T (je 16). Auf die drei häufigsten Buchstaben entfallen $41 \%$ der Gesamtmenge, auf die vier nächsten $31 \%$. Damit ist ausgeschlossen, daß Messiaen die Präparierung des Textes mit Blick auf eine von ihm beabsichtigte Veränderung der Häufigkeit bestimmter Buchstaben vorgenommen hat. 
unübersehbare Ähnlichkeiten miteinander haben. Ungeachtet der Herkunft der Töne aus den Buchstaben läßt sich die in der langage communicable verfaßte Oberstimme des 1. Satzes als Folge von Motiven darstellen, die miteinander im Sinne der entwickelnden Variation verbunden sind. Dies sei anhand des folgenden Schemas erläutert:

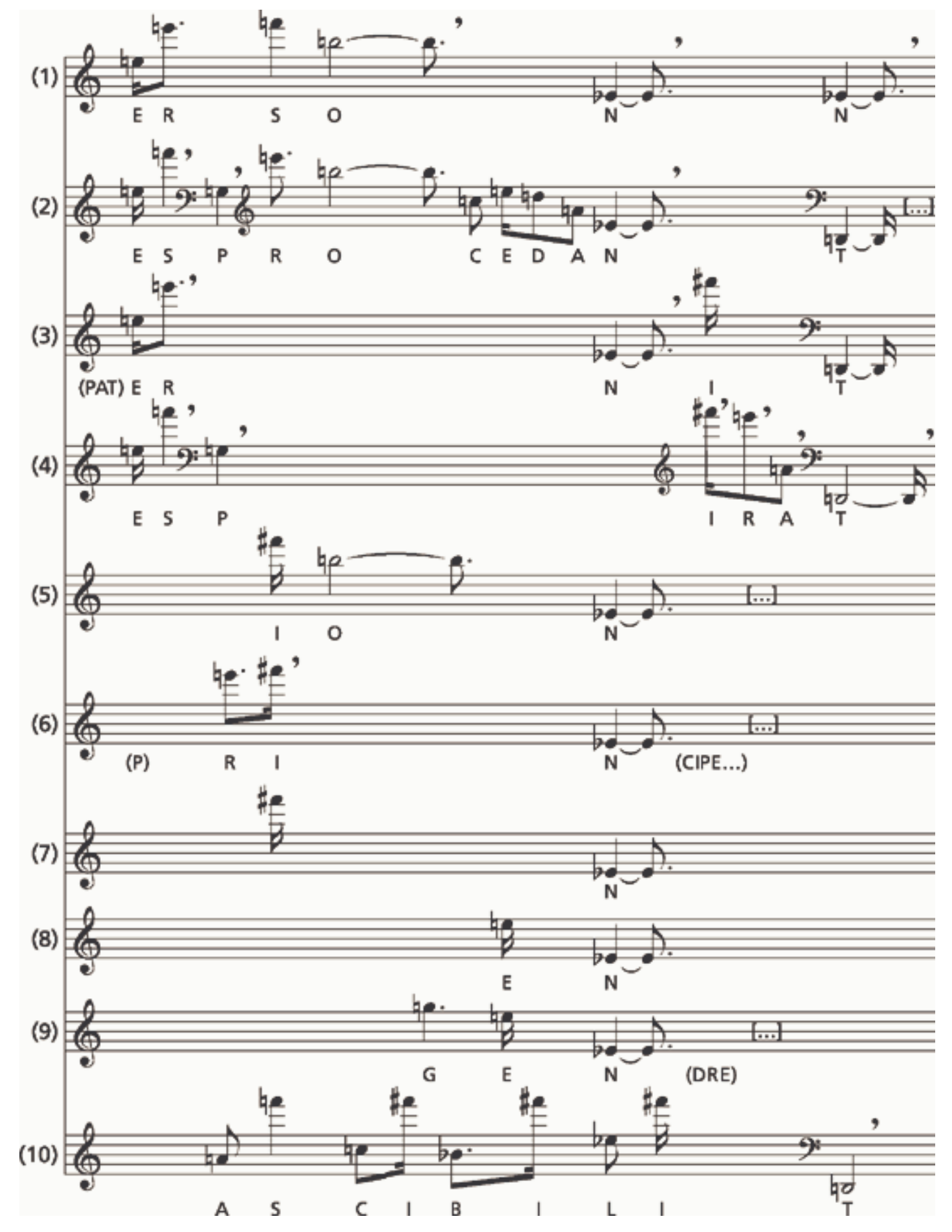


Den Zeilen 1 bis 4 des Schemas ist gemeinsam der Sprung vom e' um ungefähr eine Oktave nach oben, die von dort aus fallende Tendenz der Melodielinie sowie der Abschluß in tiefer Lage. Die Varianten der Motivanfänge basieren auf der Tatsache, daß die Tonbuchstaben $\mathrm{R}$ und $\mathrm{S}$ gewissermaßen "verwechselbar" sind, indem ihnen benachbarte Töne ähnlicher Länge zugeordnet wurden, sowie darauf, daß das I ebenfalls in der dreigestrichenen Oktave angesiedelt wurde. In Zeile 2 bleibt der Rahmen des ersten Motivabschnitts aus Zeile $1, e$ " $-h$ ' $-e s^{\prime}$, erhalten, während der zweite und dritte Ton vertauscht werden. Der Ton g wird als Variante zwischen den 2. und 3. Ton geschaltet; ebenso wird an der Stelle der ersten Zäsur der Sprung $h$ "' $-e s$ ' durch vier zusätzliche kurze Töne ausgefüllt. Die Anfänge der folgenden Zeilen 3 bis 7 lassen sich verstehen als Reduzierungen des Zeilenbeginns. Nachdem sich Zeile 3 auf drei Gerüsttöne $e^{, '}-e$,' $-e s$ ' beschränkt, greift Zeile 4 auf den Beginn von Zeile 2 zurück. Zeile 5 schließlich ersetzt die bisher aufgetretenen Anfangstöne durch ein kurzes fis',', das die bisher am Motivbeginn verwendeten Töne der dreigestrichenen Oktave an Höhe noch übertrifft. Die folgenden beiden Zeilen bilden unterschiedliche Reduzierungen von Zeile 5, die alle auf das $e s^{\prime}$ als Zielpunkt zulaufen. Zeile 8 ist eine gestauchte Fassung der vorausgehenden Zeile, die in Zeile 9 durch einen vorausgehenden Zusatzton ergänzt wird.

$$
-24-
$$

Die Varianten der Motivschlüsse beruhen auf der Ähnlichkeit der Tonbuchstaben N und $\mathrm{T}$, die beide mit relativ langen und relativ tiefen Tönen verbunden sind. Der Sprung um eine kleine None nach unten am Motivschluß von Zeile 2 läßt sich, da er die Abwärtstendenz der Linie verstärkt, als Variante der Formulierung aus Zeile 1 auffassen. Zeile 3 fügt zwischen die Schlußtöne einen kurzen Hochton ein, aus dem sich in Zeile 4 ein dreitöniges Motiv entwickelt; vor diesem entfällt der erste der beiden ursprünglichen Schlußtöne.

Aus der Vorordnung des Materials im Sinne der langage communicable ergibt sich also ein musikalisch sinnvoller und alles andere als "zufälliger" Verlauf. Daß sich die Melodieabschnitte der Oberstimme als Folge von aus einander entwickelten Motiven verstehen lassen, folgt aus der Zuordnung von Dauern und Lagen zu den häufig verwendeten Buchstaben der Textgrundlage. Die Spitzentöne R, S und I können einander ersetzen, ohne daß sich die Tendenz der Linie ändert, denn sie sind alle sehr hoch und relativ kurz. Eine vergleichbare Beziehung besteht zwischen $\mathrm{N}$ und $\mathrm{T}$, den beiden längsten und relativ tiefen Tönen. Beide eignen sich als Schlüsse eines abwärtsgerichteten Motivs, 
und die Ersetzung eines N-Schlusses durch einen T-Schluß wirkt wie eine Dehnung des Motivs. Problematisch wäre einzig die Folge T-N, die aber erfahrungsgemäß im Französischen selten auftritt.

$$
-26-
$$

Nicht in die Folge der entwickelnden Variation einzufügen scheint sich nur eine Stelle kurz vor Schluß des Abschnitts (im Schema: Zeile 10). Erhalten bleibt hier nur der äußere Umriß: sehr hohe Töne zu Beginn und die halbe Note D am Schluß. Der in den anderen Motiven anzutreffende wellenförmige Melodieverlauf wird hier jedoch ersetzt durch eine Folge von großen Intervallsprüngen. Diese ist bedingt durch die Häufigkeit des Tonbuchstaben I, der nicht nur der höchste Ton des Tonalphabets ist, sondern auch der einzige Vokal, der mit der extrem hohen Lage (dreigestrichene Oktave) verbunden wurde. Es ist anzunehmen, daß der Komponist diese Auffälligkeit bewußt als Kontrastmoment eingeplant hat.

\section{III}

$-27-$

Die Vorworte und Werkkommentare Messiaens sind keine autorisierten Deutungen und schon gar nicht wörtliche "Übersetzungen" seiner Musik. Im Gegenteil werfen sie sofern sie isoliert vom Notentext gelesen werden - kein gutes Licht auf den Komponisten, kann ihnen doch zu Recht der Vorwurf der Naivität, Ungereimtheit, Pseudowissenschaftlichkeit gemacht werden. Nicht selten suggerieren sie gar, daß beim Komponieren geradezu unkünstlerische Verfahrensweisen angewendet wurden: daß Tonfolgen nach dem Automatismus eines Tonalphabets oder entsprechend im voraus festgelegter arithmetischer Operationen abgespult werden oder daß einfach die Natur plagiiert wird. Dies sollte jedoch nicht dazu verleiten, die Verbaltexte zu ignorieren. Ohne Kenntnis der Textgrundlage und des Verfahrens der langage communicable hätte nicht nur unklar bleiben müssen, welche Intentionen der Komponist mit den Méditations verfolgt, sondern auch die Raffinesse der Materialvorordnung, wie sie sich der Analyse zeigte, ließe sich gar nicht ermessen. Zu plädieren ist für einen reflektierten Umgang mit Messiaens Texten. Wie charakteristische Werktitel oder Programme von Sinfonischen Dichtungen gehören sie zum ästhetischen Objekt, aber sie erklären und interpretieren es nicht, sondern sind selbst zu interpretieren im Zusammenhang des ganzen Werks und im Zusammenhang der Poetik des Komponisten. 
Über das "Geheimnis der Heiligen Dreieinigkeit" wird in dem uns vorliegenden Werk auf mehreren Ebenen musikalisch "meditiert". So geht es zunächst um die musikalische Umsetzung bestimmter theologischer Vorstellungen, die sich bereits auf bildliche oder symbolische Repräsentationen beziehen. Die Worte "Père des étoiles" etwa (1. Satz) werden in eine musikalische Abbildung der Sterne übertragen, "Dieu est Saint" (2. Satz) bedient sich einer gregorianischen Choralmelodie, eines "thème de couleurs" aus Messiaens Technique musical sowie einiger Vogelstimmen als Symbole. Eine zweite Form der musikalischen "Meditation" beruht auf der Erkenntnis, daß bestimmte Aspekte der Trinität aufgrund ihres Abstraktionsgrades nur mit Hilfe der Wortsprache auszudrücken sind - eine Erkenntnis, die keiner Kapitulation des Musikers vor den überlegenen Möglichkeiten der Wortsprache gleichkommt, sondern sich auf die besondere Bedeutung bezieht, die dem Wort in der christlichen Religion und Theologie beigemessen wird. Liegt somit bereits im Konzept der langage communicable eine theologische Aussage verborgen, so ist ihre Anwendung eine ästhetische Umsetzung des Begriffs "mystère": Das "Geheimnis" des Ursprungs Gottes - Thema der Textgrundlage für den ersten Satz - wird durch die Übertragung in die langage communicable und die konkrete musikalische Bearbeitung nicht aufgedeckt, sondern weiter verdunkelt. $-29-$

Gleichzeitig ist die langage communicable eine Methode, ein von subjektiver "Inspiration" und menschlichem Willen freies musikalisches Material herzustellen. Für seine auf Vogelstimmen beruhenden Werke hat der Komponist dieses Motiv in aller Deutlichkeit formuliert:

“Aber die Inspiration ist keine Frucht des Willens. Und wenn alles verloren scheint, wenn man keinen Weg mehr findet, wenn man wirklich nichts mehr zu sagen weiß (und dies kommt leider am häufigsten vor!), an welchen Meister soll man sich wenden, welchen 'Daimon' beschwören, um aus diesem Abgrund herauszufinden? Angesichts so vieler entgegengesetzter Schulen, überlebter Stile und sich widersprechender Idiome gibt es keine menschliche Musik, die dem Verzweifelten Vertrauen einflößen könnte. Da greifen die Stimmen der unendlichen Natur ein. "20

$\overline{20}$ Olivier Messiaen, Vortrag in Brüssel, in: Musik-Konzepte 28. Olivier Messiaen, hrsg. von HeinzKlaus Metzger und Rainer Riehn, München 1982, S. 5. 
So romantisch seine Worte gelegentlich erscheinen mögen - aufgrund seines musikalischen Denkens gehört Messiaen zur musikalischen Moderne, die er umgekehrt mit seinem Denken selbst stark geprägt hat. Das Verfahren der langage communicable kommt keinem Automatismus gleich, sondern es ergibt ein sorgfältig auf seine musikalischen Konsequenzen befragtes vorgeordnetes Material, dessen Verwendung zu einem künstlerisch überzeugenden Ergebnis führt. Und dieses Ergebnis ist es, das die Méditations sur le mystère de la Sainte Trinité, ungeachtet der logischen Schwächen ihres Vorwortes, zu einem der großen geistlichen Werke des 20. Jahrhunderts macht. 